\title{
Effectiveness of an internet provided behavioral weight loss intervention delivered to breast cancer survivors
}

\begin{abstract}
Purpose: Excess weight is associated with negative outcomes among breast cancer survivors. Our primary objective was to pilot test the value of an internet delivered behavioral weight loss (BWL) intervention for breast cancer survivors.
\end{abstract}

Methods: Stage 0-III breast cancer survivors with excess weight who did (CHEMO) and did not receive chemotherapy (NO CHEMO) were recruited to participate. The 6month BWL intervention included behavior modification strategies, calorie reduction and an increase in moderate exercise. The primary outcome was change in anthropometrics. Secondary outcomes were change in food intake and physical activity.

Results: Fifty women were recruited (25 who received chemotherapy; 25 who did not receive chemotherapy) and 34 completed post testing. The results from both an intentto-treat analysis and analyzing including only subjects who completed the intervention identified that both the CHEMO and NO CHEMO groups lost significant weight. Participants in the CHEMO and NO CHEMO groups who completed the intervention lost $4.5 \%$ and $7.5 \%$ of baseline body weight respectively. The NO CHEMO group lost a significant percent of their baseline body fat while the CHEMO group did not. The intervention resulted in a significant fat free mass loss for the CHEMO group, while fat free mass was preserved in the NO CHEMO group. The calorie deficit for the NO CHEMO group was significant while the CHEMO group did not achieve a significant reduction in calories consumed. No exercise parameter changed in either group.

Conclusion: Individuals with breast cancer experience significant weight loss with an internet delivered intervention. Women who received chemotherapy lost less weight, less body fat and more fat free mass than survivors who did not receive chemotherapy. Despite variable success between groups, an internet based BWL program results in weight loss in a breast cancer survivor population.

Keywords: breast cancer survivor, weight loss
Volume 2 Issue 3 - 2015

\author{
Kim Dittus L,' Janice Bunn Y,2 Nathan \\ Kokinda $D,{ }^{3}$ Karen Wilson $M,{ }^{4}$ Alan Howard \\ $B,{ }^{2}$ Jean Harvey $R^{5}$ \\ 'Department of Internal Medicine, University of Vermont, \\ USA \\ ${ }^{2}$ Department of Mathematics and Statistics, University of \\ Vermont, USA \\ ${ }^{3}$ Department of Rehabilitation and Movement Science, \\ University of Vermont, USA \\ ${ }^{4}$ UVM Cancer Center, University of Vermont, USA \\ ${ }^{5}$ Department of Nutrition and Food Sciences, University of \\ Vermont, USA
}

Correspondence: Kim Dittus, Department of Internal Medicine, University of Vermont, Given E-214, 89 Beaumont Ave, Burlington Vermont 05405, USA, Tel 8026565487, Fax 8026565493,Email kim.dittus@vtmednet.org

Received: April 20, 2015 | Published: May 07, 2015
Abbreviations: BWL, behavioral weight loss; BMI, body mass index; AEE, activity-related energy expenditure; ANCOVA, analysis of covariance; SD, standard deviation; GED, general equivalency diploma; SEM, standard error of the mean

\section{Introduction}

The percentage of overweight or obese breast cancer survivors is $66 \%$ mirroring the general population..$^{1-3}$ Excess weight at diagnosis is often compounded by weight gain post therapy, especially among individuals who received chemotherapy ${ }^{3,4}$ and exceeds the $3-5 \mathrm{~kg}$ per decade gain observed in the general population. ${ }^{5}$ Additionally, the weight gained during treatment for breast cancer often results in increased body fat and loss of lean mass, or sarcopenia. ${ }^{6-8}$

Breast cancer survivors with excess weight are at increased risk of recurrence as well as disease specific and overall mortality. ${ }^{9-13}$ The increased overall mortality among those with excess weight is a particular concern in women with early stage breast cancer who are likely cured of their breast cancer but may be at increased risk for chronic diseases, such as coronary heart disease. Because of the negative consequences associated with excess weight, the American
Cancer Society has called for weight loss to be standard of care for overweight and obese breast cancer survivors. ${ }^{14}$

Successful weight loss interventions emphasize behavior modification, energy restriction and increased physical activity. ${ }^{15,16}$ Among the general population, as well as those with and at risk for diabetes, this approach results in $7-10 \%$ weight loss. ${ }^{17,18}$ Behaviorally based weight loss interventions for breast cancer survivors have resulted in weight losses ranging from $2.4 \%-13.9 \% .{ }^{19-25}$ However, dissemination of behaviorally based weight loss interventions for cancer survivors will require distantly delivered options. Technologies such as the Internet can be used to foster the intense monitoring and interactions needed for weight loss and has been successful among the general population. ${ }^{26}$ However, an internet delivered intervention has not been tested with breast cancer survivors. Therefore, the aim of this study was to pilot test an Internet delivered behavioral weight loss (BWL) intervention among breast cancer survivors who did and did not receive chemotherapy. Primary outcomes were weight loss and anthropometric change. Secondary outcomes included change in energy balance parameters. We also explored difference in response to the intervention between women who did and did not receive chemotherapy. 


\section{Methods}

\section{Design}

The efficacy of a 6month, Internet-based, BWL intervention was tested with a two arm pre-post test study design. Breast cancer survivors who received (CHEMO) and did not receive chemotherapy (NO CHEMO) during their initial cancer therapy were recruited. Study measurements were obtained at baseline and 6months. The study was approved by the Institutional Review Board at the University of Vermont and informed consent was obtained from all participants. Power analysis suggested that 17 women per group would provide $90 \%$ power to detect a within group weight loss of $6.95 \pm 18.2 \mathrm{~kg}$ after six months, a tracking correlation of 0.90 and alpha of $0.05 .{ }^{27}$ A $20 \%$ attrition rate was assumed.

\section{Study population}

Potential participants included women with non-metastatic breast cancer completing initial oncologic therapy at least 6 weeks prior to study initiation. Oncologic intervention included surgery \pm radiation and chemotherapy depending on the subject group. Eligible subjects were postmenopausal, age 65 or younger, had a BMI between 26 and $50 \mathrm{~kg} / \mathrm{m}^{2}$ and access to a computer with internet service. Postmenopausal status was defined as last menstrual period at least 12 months prior to study enrollment. Women with medical conditions limiting food choice or ability to walk for exercise were excluded from participation.

\section{Weight loss intervention}

The weight loss intervention was a 6month (24week), behavioral, weight loss program delivered on-line. Intervention components included calorie restriction, physical activity and behavioral modification. ${ }^{28,29}$ Self-monitoring skills are key weight loss behaviors and participants were instructed to record their dietary intake, minutes of physical activity and weight daily in an online journal. Other behavioral strategies included stimulus control, problem solving, social support and relapse prevention. A 60 minute, on-line synchronous group meeting was held once a week and was led by an interventionist with experience in promoting lifestyle change. Lessons could be accessed ahead of the group meeting. Other educational material was available on-line and could be accessed at any time. Participants were asked to reduce their energy intake by up to $1000 \mathrm{kcal} /$ day but not lower than $1200 \mathrm{kcal} /$ day. Individual goals were determined by multiplying baseline weight in pounds by 12 (an estimate of current calorie consumption) and subtracting 1000calories. This reduction is known to produce a weight loss of approximately $1-2 \mathrm{lbs} /$ week. $^{30,31}$ Moderate intensity aerobic exercise was increased gradually from 50 minutes/week $(250 \mathrm{kcal})$ to a goal of 400 minutes a week $(2,000 \mathrm{kcal}) .^{32,33}$ To achieve this goal, exercise was prescribed for at least 5 days/week. Brisk walking was the primary mode of activity.

\section{Study measures}

Weight and height were obtained on a calibrated digital scale (Scale-tronix) and a wall mounted Holtain stadiometer respectively. BMI was calculated as weight $(\mathrm{kg}) / \mathrm{height}^{2}(\mathrm{~m})$. Body composition, including total fat mass and fat free mass was assessed for the breast cancer participants using a dual energy X-ray absorptiometry using a Lunar Prodigy densitometer (Lunar Co, Madison WI).

Estimates of energy and fat intake were determined using three 24-hour recalls collected at baseline and post intervention, using the NCI Automated self-administered 24hour recall (ASA24). ${ }^{34}$ ASA24 uses computer technology to guide participants toward accurate assessment of portion size and food content. The list from which respondents select their food intakes was the USDA's most current Food and Nutrient Database for Dietary Studies database. Activityrelated energy expenditure (AEE) and physical activity duration was measured using Body Media ${ }^{\circledR}$ Body Monitoring System accelerometer worn for 7days. AEE includes calories expended in activities of $\geq 3$ METS. Physical activity duration included the minutes of activity performed at $\geq 3$ METS.

Compliance was assessed by attrition and adherence. Intervention attrition was the percent of individuals who discontinued the intervention after they joined the group. Adherence was determined by the number of on-line group meetings attended. Self-monitoring of food intake or "journaling" was tracked by the interventionist leading the group. A participant was considered to have participated in weekly self-monitoring if they "journaled" any food intake for the 7days prior to the weekly group meeting. A total of 24 group sessions and weekly journaling opportunities were available for each participant.

\section{Statistics}

Descriptive statistics for demographic and baseline variables were calculated. Results are presented as mean \pm SD or SEM depending on the variable. The variables were approximately normally distributed. Baseline differences between intervention groups and between completers and non-completers were assessed using $t$ tests for continuous variables. The percent of participants achieving $7 \%$ weight loss, the goal of large weight loss interventions ${ }^{28,29}$ and $5 \%$ weight loss, the lower threshold of loss for physical health benefits, ${ }^{35,36}$ was determined.

A two-group repeated measures analysis of variance using linear mixed models, with chemotherapy/no chemotherapy as the betweengroups factor and time as the repeating factor was used. Any significant group by time interaction was followed by post hoc analyses to examine differences in treatment means at the post intervention time point. We adjusted for age and current endocrine therapy. Two sets of analysis were performed on primary measures:

1) An intent to treat analysis including all participants with baseline data, with missing values imputed by carrying forward baseline values and

2) A completers analysis that included only participants who completed baseline and post intervention testing.

For secondary measures, analysis was performed only for individuals completing the intervention. An analysis of covariance (ANCOVA) was used to determine differences in percent of weight lost between the CHEMO/NO CHEMO groups. Exploratory analysis to identify between group differences was performed for those completing the intervention. All statistical analysis was performed using the SAS System for Windows, version 9.4.

\section{Results}

Fifty participants were recruited; baseline data was obtained on 48 and 34 completed the intervention. Figure 1 represents the flow diagram for study participation. The average age for the breast cancer survivors receiving chemotherapy was younger than those who did not receive chemotherapy (Table 1). The marital status and education levels were similar between the two groups. All women were Caucasian. The average baseline BMI for both groups was similar. The mean interval from breast cancer diagnosis to study entry was $30.8 \pm 16$ months and $32.4 \pm 22.5$ months for the CHEMO and NO CHEMO group respectively (range $9-110$ months). As expected, the 
CHEMO group included more participants with higher stage breast cancer. A greater proportion of those in the NO CHEMO group received endocrine therapy.

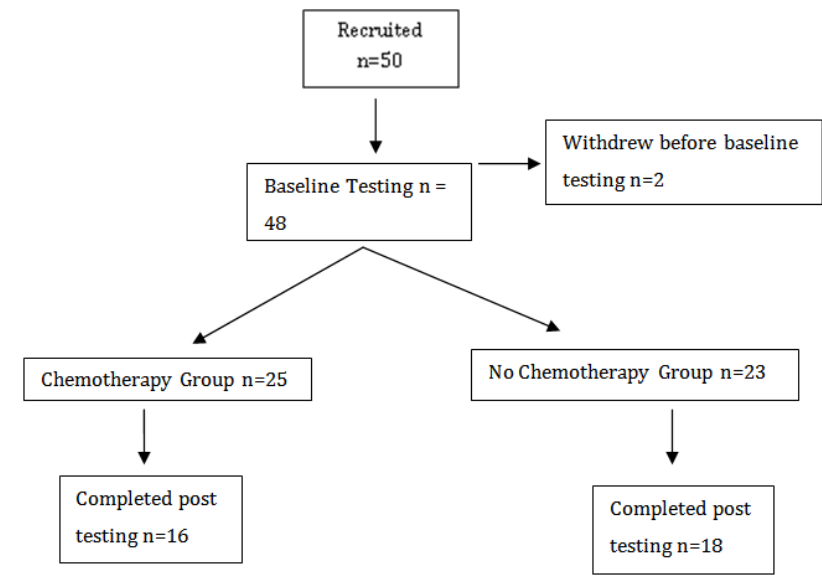

Figure I Flow of participants.

\section{Primary outcomes}

Both intent to treat analysis and analysis of only those completing the intervention identified a significant weight loss and corresponding decrease in BMI within the CHEMO and NO CHEMO groups (Table 2).The change in anthropometric measures was not significantly different between the two breast cancer groups (Table 3) but positive anthropometric change favored the NO CHEMO group for all measures.

The NO CHEMO group also achieved a significant reduction in percent body fat while maintaining fat free mass using intent to treat approach and analysis of completers. However, the percent body fat lost using intent to treat analysis was not significant for the CHEMO group but they did lose a significant amount of fat free mass. The fat free mass of individuals in the NO CHEMO group was preserved. The proportion of individuals completing the intervention that achieved the goal of 7\% weight loss was similar between the CHEMO and NO CHEMO groups at $37.5 \%$ and $38.9 \%$ respectively. However, a greater proportion of the NO CHEMO group completers lost at least $5 \%$ of baseline body weight.

Table I Baseline characteristics of participants

\begin{tabular}{|c|c|c|}
\hline & $\operatorname{CHEMO}(n=25)$ & NO CHEMO $(n=23)$ \\
\hline Age, years (mean $\pm S D$ ) & $52.6 \pm 6.8$ & $57.5 \pm 4.4$ \\
\hline \multicolumn{3}{|l|}{ Education } \\
\hline HS grad/GED & I (4\%) & I (4.4\%) \\
\hline Some College & $6(24 \%)$ & $5(21.7 \%)$ \\
\hline College Graduate & $8(32 \%)$ & $9(39.1 \%)$ \\
\hline Post Graduate & $10(40 \%)$ & $8(34.8 \%)$ \\
\hline \multicolumn{3}{|l|}{ Work for Pay } \\
\hline Full-Time & $15(60 \%)$ & II (52\%) \\
\hline Part-Time & $5(20 \%)$ & $5(24 \%)$ \\
\hline Not Employed & $5(20 \%)$ & $5(25 \%)$ \\
\hline Married & $20(80 \%)$ & $16(70 \%)$ \\
\hline Baseline BMI $\left(\mathrm{kg} / \mathrm{m}^{2}\right)($ mean $\pm \mathrm{SD})$ & $33.4 \pm 5.3$ & $33.5 \pm 6.4$ \\
\hline \multicolumn{3}{|l|}{ Stage (n) } \\
\hline 0 & 0 & $5(22 \%)$ \\
\hline I & $7(28 \%)$ & $18(78 \%)$ \\
\hline ॥ & II (44\%) & 0 \\
\hline III & $7(28 \%)$ & 0 \\
\hline Receipt of Endocrine Therapy ( $n$ ) & 18 (72\%) & $22(96 \%)$ \\
\hline Time from Diagnosis, Months (mean $\pm S D$ ) & $30.8 \pm 16.0$ & $32.4 \pm 22.5$ \\
\hline
\end{tabular}

$\mathrm{SD}$, standard deviation; GED, general equivalency diploma; BMI, body mass index 
Table 2 Anthropometric and weight loss change among participants controlled for age and endocrine therapy for breast cancer survivors

\begin{tabular}{|c|c|c|c|c|}
\hline & \multicolumn{2}{|c|}{ Completers } & \multicolumn{2}{|c|}{ Intent to treat } \\
\hline & CHEMO & NO CHEMO & CHEMO & NO CHEMO \\
\hline $\mathbf{N}$ & 16 & 18 & 25 & 23 \\
\hline & $4.7 \pm 1.2$ & $6.0 \pm 1.2$ & $3.0 \pm 0.9$ & $4.7 \pm 1.0$ \\
\hline \multicolumn{5}{|l|}{ Weight Loss, kg } \\
\hline & $\mathrm{P}=0.0004$ & $p<0.0001$ & $\mathrm{P}=0.002$ & $P<0.0001$ \\
\hline & $4.5 \pm 1.6$ & $7.5 \pm 1.5$ & $2.9 \pm 1.2$ & $5.8 \pm 1.2$ \\
\hline \multicolumn{5}{|l|}{$\%$ Weight lost } \\
\hline & $p=0.009$ & $p<0.0001$ & $p=0.02$ & $\mathrm{P}<0.0001$ \\
\hline$\%$ losing $\geq 7 \%$ & $37.50 \%$ & $38.90 \%$ & $24.00 \%$ & $30.40 \%$ \\
\hline$\%$ losing $\geq 5 \%$ & $50 \%$ & $72.20 \%$ & $32.00 \%$ & $56.50 \%$ \\
\hline & $1.7 \pm 0.5$ & $2.3 \pm 0.4$ & $I . I \pm 0.4$ & $1.8 \pm 0.4$ \\
\hline \multicolumn{5}{|c|}{ BMI Loss, kg/m² } \\
\hline & $p=0.0005$ & $p<0.0001$ & $p=0.002$ & $\mathrm{p}<0.0001$ \\
\hline & $1.6 \pm 0.7$ & $3.1 \pm 0.7$ & $1.0 \pm 0.5$ & $2.4 \pm 0.5$ \\
\hline \multicolumn{5}{|c|}{ Body Fat Loss, \% } \\
\hline & $p=0.03$ & $\mathrm{p}<0.0001$ & $p=0.06$ & $p<0.0001$ \\
\hline & $3.5 \pm 1.0$ & $5.1 \pm 1.0$ & $2.2 \pm 0.8$ & $3.9 \pm 0.8$ \\
\hline \multicolumn{5}{|c|}{ Fat Mass Loss, kg } \\
\hline & $p=0.002$ & $\mathrm{p}<0.000 \mathrm{I}$ & $p=0.01$ & $P<0.0001$ \\
\hline & $1.4 \pm 0.4$ & $0.8 \pm 0.4$ & $0.9 \pm 0.3$ & $0.6 \pm 0.3$ \\
\hline \multicolumn{5}{|c|}{ Fat Free Mass Loss, kg } \\
\hline & $\mathrm{p}=0.00 \mathrm{I}$ & $p=0.06$ & $p=0.003$ & $p=0.06$ \\
\hline
\end{tabular}

Data are given as means \pm SEM, standard error of the mean; BMI, body mass index

Table 3 Between groups comparisons for participants completing the intervention controlled for age and endocrine therapy

\begin{tabular}{|c|c|c|c|}
\hline Variable & CHEMO $n$ = 16 & NO CHEMO $n=18$ & p Value \\
\hline Weight Loss, kg & $4.7 \pm 1.2$ & $6.0 \pm 1.2$ & 0.43 \\
\hline$\%$ Weight Lost & $4.5 \pm 1.6$ & $7.5 \pm 1.5$ & 0.24 \\
\hline BMI Loss, kg/m² & $1.7 \pm 0.5$ & $2.3 \pm 0.4$ & 0.38 \\
\hline \% Body Fat Decline & $1.6 \pm 0.7$ & $3.1 \pm 0.7$ & 0.11 \\
\hline Fat Mass Loss, kg & $3.5 \pm 1.0$ & $5.1 \pm 1.0$ & 0.28 \\
\hline Fat Free Mass Loss, kg & $1.4 \pm 0.4$ & $0.8 \pm 0.4$ & 0.25 \\
\hline
\end{tabular}

Data are given as means \pm SEM, standard error of the mean; BMI, body mass index

Secondary Outcomes NO CHEMO participants completing the intervention experienced a statistically significant decrease in calorie intake while there was no decrease in calories for the CHEMO group (Table 4). There were no significant changes in AEE or moderate physical activity duration. The attrition rate of was $36 \%$ for the CHEMO group and $22 \%$ for the NO CHEMO group (Table 5). The NO CHEMO group had a higher attendance and more frequent journaling. 
Table 4 Diet and exercise change from baseline to intervention completion for participants completing the intervention controlled for age and endocrine therapy

\begin{tabular}{|c|c|c|}
\hline Variable & CHEMO $(n=16)$ & NO CHEMO $(n=\mid 8)$ \\
\hline \multicolumn{3}{|c|}{ K cal Intake } \\
\hline Baseline & $2093 \pm 132$ & $1901 \pm 139$ \\
\hline 6month & $1892 \pm 109$ & $1610 \pm 144$ \\
\hline Change & $-103.3 \pm 110.4$ & -283.3 \\
\hline$P$ value & 0.36 & 0.02 \\
\hline \multicolumn{3}{|c|}{ AEE, K cals/week } \\
\hline Baseline & $290 \pm 38$ & $338 \pm 50$ \\
\hline 6month & $270 \pm 41$ & $345 \pm 68$ \\
\hline Change & -20 & $+7.4 \pm 75.3$ \\
\hline $\mathrm{P}$ value & 0.25 & 0.62 \\
\hline \multicolumn{3}{|c|}{ Physical Activity Duration min/week } \\
\hline Baseline & $5 I \pm 7$ & $61 \pm 9$ \\
\hline 6month & $4 \pm 7$ & $68 \pm 13$ \\
\hline Change & $-14.0 \pm 17.6$ & $+9.4 \pm 16.3$ \\
\hline$P$ value & 0.43 & 0.57 \\
\hline
\end{tabular}

Data are given as mean \pm SEM: Standard Error of the Mean, $\mathrm{p}$ values represents the differences in the change scores of baseline-6mo. AEE, active energy expenditure

Table 5 Compliance Parameters for participants completing the intervention

\begin{tabular}{lll}
\hline Variable & CHEMO & NO CHEMO \\
\hline Attrition (n) & $9(36 \%)$ & $5(22 \%)$ \\
Attendance & $10.5 \pm 8.0(44 \%)$ & $14.1 \pm 5.9(58 \%)$ \\
Journaling & $9.9 \pm 9.2(41 \%)$ & $12.9 \pm 9.1(54 \%)$ \\
\hline
\end{tabular}

Data are given as means \pm SD: Standard Deviation

\section{Discussion}

The American Cancer Society encourages weight loss for overweight cancer survivors..$^{14}$ Behavioral weight loss interventions that modify behavior, reduce calories and increase physical activity are the gold standard for the general population..$^{15,16}$ However, in person group interventions are unrealistic for a population of cancer survivors that live distant from sites where interventions are delivered. Ours is the first intervention to document successful weight loss for breast cancer survivors provided an on-line BWL approach. Weight loss and BMI results were significant whether analysis used an intentto-complete approach or included only individuals completing the program. The intervention provided to the breast cancer survivors for this study has been successful in a general population from Arkansas and Vermont. ${ }^{27}$ The weight loss success of the breast cancer survivors who did not receive chemotherapy (6.0 \pm 1.2$)$ was similar to the general population of post-menopausal women from Vermont $(6.6 \pm 0.9)$ who received the same intervention. ${ }^{27}$
A goal of $7 \%$ of baseline weight loss has been used for large weight loss interventions ${ }^{28,29}$ and a $5 \%$ weight loss results in physiologic improvement. ${ }^{35,36}$ Overall, individuals completing the intervention who did not receive chemotherapy lost over $7 \%$ of their body weight, a percent of weight loss that mirrored that reported in the general population from Vermont (7.7\%) receiving the same intervention. ${ }^{37}$ However, those receiving chemotherapy lost less than $5 \%$ of their baseline weight. A similar percent of both breast cancer groups achieved the 7\% goal, but a larger percent of the NO CHEMO group achieved $5 \%$ loss of baseline weight $(72.2 \%$ vs. $50 \%)$.

This is the first weight loss intervention to compare weight loss success breast cancer survivors who did and did not receive chemotherapy. Differences were identified in how the breast cancer groups responded to the intervention. In particular individuals who received chemotherapy were less successful. They lost $1.7 \mathrm{~kg}$ less weight than the NO CHEMO group using intent to treat analysis. The percent weight loss and proportion losing at least $5 \%$ of baseline body weight was also less for the CHEMO group. The CHEMO group did not achieve a significant reduction in percent body fat but did experience a significant decline in fat free mass compared to their baseline. Prior research identifies that individuals who receive chemotherapy tend to gain weight and lose muscle mass. ${ }^{6-8}$ Translational studies are beginning to identify impacts of chemotherapy on skeletal muscle. ${ }^{37}$ Perhaps chemotherapy creates muscle metabolism change that makes maintaining muscle mass more challenging. Despite within group differences in response to the intervention for the two group, the between group differences were not statistically significant. With less than 20 individuals in each group, the lack of significance in weight lost may be due to insufficient power. A larger study would be required to further explore whether cancer survivors who receive chemotherapy have greater difficulty with weight loss.

Weight loss requires an energy balance deficit created by decreased caloric intake and increased energy expenditure. The NO CHEMO group achieved a significant decrease in caloric intake. The caloric deficit for women who received chemotherapy was not significant and may have contributed to less weight loss. AEE and time spent in moderate intensity physical activity did not change as a result of the intervention for either group. An on-line BWL intervention could be modified to emphasize the value of exercise by requiring greater exercise self-monitoring and interventionist feedback. Incorporating tracking devices such as those available commercially may also improve exercise compliance.

The average attrition rate for intense weight loss interventions is $37 \%{ }^{38}$ and varies from $10-80 \%{ }^{39}$ The attrition rate for the breast cancer survivors in the current study was $36 \%$ for individuals receiving chemotherapy and $22 \%$ for those who did not, both somewhat higher than expected. We do not believe the on-line delivery contributed to attrition as no differences in attrition rates were identified in the only randomized comparison of in-person vs. on line behavioral weight loss interventions for a general population. ${ }^{27}$ The NO CHEMO group attended more group sessions and "journaled" more consistently than the CHEMO group, activities that likely contributed to their improved weight loss success. Perhaps individuals who receive chemotherapy have additional barriers to compliance and success with weight loss interventions. Receipt of chemotherapy has been associated with declines in executive function. ${ }^{40}$ Executive function skills are important for complying with the complex changes need to lose weight and may have contributed to difficulty completing the 
multiple tasks of a BWL intervention. Exploration of contributors and barriers to compliance among cancer survivors receiving different types of oncologic interventions would be useful for designing future weight loss interventions.

Strengths of our pilot intervention included the successful demonstration that breast cancer survivors can achieve weight loss with an internet delivered BWL intervention. Additionally, this is the first study to explore differences in efficacy of weight loss interventions provided to breast cancer survivors who received different oncologic interventions. The study was limited to postmenopausal breast cancer survivors. Research among breast cancer survivors identifies that onset of menopause predicts weight gain. ${ }^{41}$ In addition to the study not being powered to identify differences in breast cancer survivors who did and did not receive chemotherapy and the higher than expected attrition rate mentioned above, other limitations include the inherent difficulties in accurately measuring diet and exercise which are common to weight loss intervention studies.

\section{Conclusion}

An internet delivered BWL intervention results in significant weight loss and would be an appropriate application for bringing weight loss interventions to larger numbers of breast cancer survivors. Individuals who did not receive chemotherapy appear to experience weight loss equivalent to women without cancer receiving the same intervention. An internet delivered BWL intervention appears to be less effective for breast cancer survivors receiving chemotherapy. The difference in response to weight loss between survivors receiving different oncologic interventions requires further study. Overall, calorie reduction is achieved with the BWL intervention; however, participants did not increase the time spent in moderate or vigorous physical activity. Future interventions should emphasize strategies to reach exercise goals. The variability in weight lost and the attrition rates between the two breast cancer groups suggests that a one size fits all approach is less effective than desired. Ideally, future weight loss interventions would focus on identifying predictors of attrition and successful weight loss in order to improve our ability to tailor interventions for breast cancer survivors and maximize weight loss success.

\section{Acknowledgements}

None.

\section{Conflict of interest}

The author declares no conflict of interest.

\section{References}

1. Flegal KM, Carroll MD, Ogden CL, et al. Prevalence and trends in obesity among US Adults, 1999-2008. JAMA. 2010;303(3):235-241.

2. Dawood S, Broglio K, Gonzalez-Angulo AM, et al. Prognostic value of body mass index in locally advanced breast cancer. Clin Cancer Res. 2008;14(6):1718-1725.

3. Vance V, Mourtzakis M, McCargar L, et al. Weight gain in breast cancer survivors: prevalence, pattern and health consequences. Obes Rev. 2010;12(4):282-294.

4. Heideman WH, Russell NS, Gundy C, et al. The frequency, magnitude and timing of post-diagnosis body weight gain in Dutch breast cancer survivors. Eur J Cancer. 2009;45(1):119-126.
5. Sternfeld B, Wang H, Quesenberry CP, et al. Physical activity and changes in weight and waist circumference in midlife women: Findings from the study of women's health across the nation. Am J Epidemiol. 2004;160(9):912-922.

6. Freedman RJ, Aziz N, Albanes D, et al. Weight and body composition changes during and after adjuvant chemotherapy in women with breast cancer. J Clin Endocrinol Metab. 2004;89(5):2248-2253.

7. Harvie MN, Campbell IT, Baildam A, et al. Energy balance in early breast cancer patients receiving adjuvant chemotherapy. Breast Cancer Res Treat. 2004;83(3):201-210.

8. Cheney CL, Mahloch J, Freeny P. Computerized tomography assessment of women with weight changes associated with adjuvant treatment for breast cancer. Am J Clin Nutr. 1997;66(1):141-146.

9. Ewertz M, Jensen MB, Gunnarsdottir KA, et al. Effect of obesity on prognosis after early-stage breast cancer. J Clin Oncol. 2011;29(1):2532 .

10. Protani M, Coory M, Martin JH. Effect of obesity on survival of women with breast cancer: systematic review and meta-analysis. Breast Cancer Res Treat. 2010;123(3):627-635.

11. Barnett GC, Shah M, Redman K, et al. Risk factors for the incidence of breast cancer: Do they affect survival from the disease? J Clin Oncol. 2008;26(20):3310-3316.

12. Reeves GK, Pirie K, Beral V, et al. Cancer incidence and mortality in relation to body mass index in the Million Women Study: cohort study. BMJ. 2007;335(7630):1134-1139.

13. Kroenke $\mathrm{CH}$, Chen WY, Rosner B, et al. Weight, weight gain and survival after breast cancer diagnosis. J Clin Oncol. 2005;23(7):1370-1378.

14. Rock CL, Doyle C, Demark-Wahnefried W, et al. Nutrition and physical activity guidelines for cancer survivors. CA Cancer J Clin. 2012;62(4):243-274.

15. Wadden TA, Foster GD. Behavioral treatment of obesity. Med Clin North Am. 2000;84(2):441-461.

16. Grave DR, Centis E, Marzocchi R, et al. Major factors for facilitating change in behavioral strategies to reduce obesity. Psychol Res Behav Manage. 2013;6:101-110.

17. Courioni CC, Lourenco PM. Long-term weight loss after diet and exercise: a systematic review. Int J Obes (Lond). 2005;29(10):11681174.

18. Wadden TA, Butryn ML, Wilson C. Lifestyle modification for the management of obesity. Gastroenterology. 2007;132(6):2226-2238.

19. Campbell KL, Van Patten CL, Neil SE, et al. Feasibility of a lifestyle intervention on body weight and serum biomarkers in breast cancer survivors with overweight and obesity. J Acad Nutr Diet. 2012;112(4):559-567.

20. Harris MN, Swift DL, Myers VH, et al. Cancer survival through lifestyle change (CASTLE): a pilot study of weight loss. Int J Behav Med. 2013;20(3):403-412.

21. Befort CA, Klemp JR, Austin HL, et al. Outcomes of a weight loss intervention among rural breast cancer survivors. Breast Cancer Res Treat. 2012;132(2):631-639.

22. Pakiz B, Flatt SW, Bardwell WA, et al. Effects of a weight loss intervention on body mass, fitness and inflammatory biomarkers in overweight or obese breast cancer survivors. Int J Behav Med. 2011;18(4):333-341.

23. Morey MC, Snyder DC, Sloane R, et al. Effects of home-based diet and exercise on functional outcomes among older, overweight longterm cancer survivors: Renew: a randomized controlled trial. JAMA. 2009;301(18):1883-1891. 
24. Mefferd K, Nichols JF, Pakiz B, et al. A cognitive behavioral therapy intervention to promote weight loss improves body composition and blood lipid profiles among overweight breast cancer survivors. Breast Cancer Res Treat. 2007;104(2):145-152.

25. Djuric Z, DiLaura NM, Jenkins I, et al. Combining weight-loss counseling with the weight watchers plan for obese breast cancer survivors. Obes Res. 2002;10(7):657-665.

26. Wieland LS, Falzon L, Sciamanna CN, et al. Interactive computer-based interventions for weight loss or weight maintenance in overweight or obese people. Cochrane Database Syst Rev. 2012;8:CD007675.

27. Harvey-Berino J, West D, Krukowski R, et al. Internet Delivered Behavioral Obesity Treatment. Prev Med. 2010;51(2):123-128.

28. Diabetes Prevention Program Research Group. The Diabetes Prevention Program (DPP): Description of lifestyle intervention. Diabetes Care. 2002;25(12):2165-2171.

29. Wadden TA, West DS, Delahanty L, et al. The Look Ahead Study: A description of the lifestyle intervention and the evidence supporting it. Obesity. 2006;14(5):737-752.

30. National Institutes of Health. The Practical Guide: Identification Evaluation and Treatment of Overweight and Obesity in Adults. National Institutes of Health, Bethesda; 2000. p. 1-94.

31. Jeffrey RW, Wing RR, Thorson C, et al. Strengthening behavioral interventions for weight loss: a randomized trial for food provision and monetary incentives. J Consult Clin Psychol. 1993;61(6):1038-1045.

32. Jakicic JM, Winters C, Lang W, et al. Effects of intermittent exercise and use of home exercise equipment on adherence, weight loss and fitness in overweight women: A randomized trial. JAMA. 1999;282(16):1554 1560 .

33. Jakicic JM, Marcus BH, Gallagher KI, et al. Effect of exercise duration and intensity on weight loss in overweight, sedentary women: A randomized trial. JAMA. 2003;290(10):1323-1330.
34. Subar AF, Kirkpatrick SI, Mittl B, et al. The automated self-administered 24-hour dietary recall (ASA24): A resource for researchers, clinicians and educators from the National Cancer Institute. J Acad Nutr Diet. 2012;112(8):1134-1137.

35. Goldstein DJ, Rampey AH, Enas GG, et al. Fluoxetine: a randomized clinical trial in the treatment of obesity. Int J Obes Relat Metab Disord. 1994;18(3):129-135.

36. Knowler WC, Barrett-Connor E, Fowler SE, et al. Reduction in the incidence of type 2 diabetes with lifestyle intervention or metformin. $N$ Engl J Med. 2002;346(6):393-403.

37. Toth MJ, Miller MS, Callahan DM, et al. Molecular mechanisms underlying skeletal muscle weakness in human cancer: reduced myosin-actin cross-bridge formation and kinetics. $J$ Appl Physiol. 2013;114(7):858-868.

38. Fabricatore AN, Wadden TA, Moore RH, et al. Attrition from randomized controlled trials of pharmacological weight loss agents: a systematic review and analysis. Obes Rev. 2009;10(3):333-341.

39. Moroshko I, Brennan L, O Brien P. Predictors of dropout in weight loss interventions: a systematic review of the literature. Obes Rev. 2011;12(11):912-934.

40. Koppelmans V, Breteler MM, Boogerd W, et al. Late effects of adjuvant chemotherapy for adult onset non-CNS cancer; cognitive impairment, brain structure and risk of dementia. Crit Rev Oncol Hematol. 2013;88(1):87-101.

41. Goodwin PJ, Ennis M, Pritchard KI, et al. Adjuvant treatment and onse of menopause predict weight gain after breast cancer diagnosis. $J$ Clin Oncol. 1999;17(1):120-129. 